\title{
Identification and expansion of the tumorigenic lung cancer stem cell population
}

\author{
A Eramo ${ }^{1}$, F Lotti ${ }^{2}$, G Sette ${ }^{2}$, E Pilozzi ${ }^{3}$, M Biffoni ${ }^{1}$, A Di Virgilio ${ }^{4}$, C Conticello ${ }^{2}$, L Ruco ${ }^{3}$, C Peschle ${ }^{1}$ and R De Maria ${ }^{* 1}$
}

Lung carcinoma is often incurable and remains the leading cancer killer in both men and women. Recent evidence indicates that tumors contain a small population of cancer stem cells that are responsible for tumor maintenance and spreading. The identification of the tumorigenic population that sustains lung cancer may contribute significantly to the development of effective therapies. Here, we found that the tumorigenic cells in small cell and non-small cell lung cancer are a rare population of undifferentiated cells expressing CD133, an antigen present in the cell membrane of normal and cancer-primitive cells of the hematopoietic, neural, endothelial and epithelial lineages. Lung cancer $\mathrm{CD}_{133}{ }^{+}$cells were able to grow indefinitely as tumor spheres in serum-free medium containing epidermal growth factor and basic fibroblast growth factor. The injection of $10^{4}$ lung cancer $\mathrm{CD}_{133^{+}}$cells in immunocompromised mice readily generated tumor xenografts phenotypically identical to the original tumor. Upon differentiation, lung cancer $\mathrm{CD}_{133^{+}}$cells acquired the specific lineage markers, while loosing the tumorigenic potential together with $\mathrm{CD} 133$ expression. Thus, lung cancer contains a rare population of $\mathrm{CD} 133^{+}$cancer stem-like cells able to self-renew and generates an unlimited progeny of non-tumorigenic cells. Molecular and functional characterization of such a tumorigenic population may provide valuable information to be exploited in the clinical setting.

Cell Death and Differentiation (2008) 15, 504-514; doi:10.1038/sj.cdd.4402283; published online 30 November 2007

Lung cancer is the most common cause of cancer-related mortality worldwide. Four main categories of lung tumors contribute to the vast majority of cases in terms of both incidence and lethality. Small cell lung cancer (SCLC) is a neuroendocrine tumor that represents about $20 \%$ of all lung cancers, while the most common forms of the so-called nonSCLC (NSCLC) include adenocarcinoma (AC), squamous cell carcinoma (SCC) and large cell carcinoma (LCC). ${ }^{1,2}$ Despite continuous efforts to improve the therapeutic response, the overall five-year survival rate for such tumors is lower than $15 \% .^{3}$ Cancer stem cells are a rare population of undifferentiated tumorigenic cells responsible for tumor initiation, maintenance and spreading. ${ }^{4}$ These cells display unlimited proliferation potential, ability to self-renew and capacity to generate a progeny of differentiated cells that constitute the major tumor population. In light of the cancer stem cell-based model, normal stem cells might be considered as a proto-tumorigenic cells endowed with some properties typical of malignant cells, including the constitutive activation of survival pathways and the ability to proliferate indefinitely. Oncogenic mutations occurring in such a favorable background may turn the finely regulated growth potential of normal stem cells into the aberrant uncontrolled growth of cancer cells.
Cancer stem-like cells have been isolated and expanded from leukemia, ${ }^{5}$ and several solid tumors, including melanoma, breast, brain, prostate, pancreatic ${ }^{6-13}$ and colon carcinomas. ${ }^{14,15}$ These cells can be expanded in vitro as tumor spheres, while reproducing the original tumor when transplanted in immunodeficient mice. Although a universal marker for cancer stem cells has not been identified, brain, hematopoietic, prostate and colon cancer stem cells exhibit the membrane antigen CD133, whose expression is shared by normal stem cells of different lineages.

The existence of human lung cancer stem cells has not been reported yet. However, indirect evidence suggests the possible presence of cancer stem cells in pulmonary tumors. Stem-like cells have been identified in mouse lung, such as a cell population able to drive the malignant transformation in experimentally induced neoplasia. ${ }^{16}$ Moreover, human lung tumors sometimes show phenotypic heterogeneity, suggesting that they may originate from a multipotent cell. ${ }^{17}$

Normal lung tissue is composed by a variety of cell types, such as basal mucous secretory cells of the trachea and bronchi, Clara cells of bronchioles, type 1 and type 2 pneumocytes of alveoli. These mature cells derive from the differentiation of lineage-restricted lung progenitor cells, which in turn originate from undifferentiated multipotent lung

\footnotetext{
${ }^{1}$ Department of Hematology, Oncology and Molecular Medicine, Istituto Superiore di Sanità, Rome, Italy; ${ }^{2}$ Department of Experimental Oncology, Mediterranean Institute of Oncology, Catania, Italy; ${ }^{3}$ Department of Experimental Medicine, Sant'Andrea Hospital, University 'La Sapienza', Rome, Italy and ${ }^{4}$ Service for Biotechnology and Animal Welfare, Istituto Superiore di Sanità, Rome, Italy

*Corresponding author: R De Maria, Department of Hematology, Oncology and Molecular Medicine, Istituto Superiore di Sanità, viale Regina Elena 299, Rome 00161, Italy. Tel: + 39 0649903121, Fax: + 390649387087 , E-mail: demaria@iss.it

Keywords: cancer stem cells; stem cell markers; lung cancer; CD133; pulmonary stem cells; tumor sphere

Abbreviations: AC, adenocarcinoma; APC, allophycocyanin; BCRP1, breast cancer resistance protein1; CC-10, Clara cell protein; CEA, carcinoembryonic antigen; ChrA, chromogranine A; CKs, cytokeratins; EGF, epidermal growth factor; Ep-CAM, epithelial cell adhesion molecule; FACS, fluorescence-activated cell sorting; FGF, fibroblast growth factor; FITC, fluorescein isothiocyanate; HMW-CKs, high molecular weight cytokeratins; LCC, large cell carcinoma; LCNEC, large cell neuroendocrine carcinoma; MTT, 3-(4,5-dimethyl-2-thiazolyl)-2,5-diphenyl 2H-tetrazolium bromide; N-CAM, neural cell adhesion molecule; NSCLC, non-small cell lung cancer; PE, phycoerythrin; SCC, squamous cell carcinoma; SCID, severe combined immunodeficiency; SCLC, small cell lung cancer; SP-C, surfactant protein C

Received 03.5.07; revised 25.10.07; accepted 25.10.07; Edited by RA Knight; published online 30.11 .07
} 
stem cells. ${ }^{18}$ Multipotent, long-lived cells have been identified throughout the airways and give rise to both transiently amplifying and terminally differentiated daughter cells. Like stem cells of other tissues, lung stem cells are responsible for local tissue maintenance and injury repair. ${ }^{19}$ Some reports have described lung stem cells as cells expressing antigens typical of undifferentiated cells, such as CD34 and breast cancer resistance protein1 (BCRP1). ${ }^{20,21}$ However, whether lung cancer might derive from the transformation of undifferentiated or differentiated cells remains to be elucidated. We therefore investigated the possibility of isolating, expanding and characterizing tumorigenic cancer stem cells from the major lung tumors.

\section{Results}

CD133-expressing tumor cells are present within lung tumors with variable frequency. Several solid tumors contain $\mathrm{CD}_{133^{+}}$tumorigenic cancer stem cells, including glioblastoma, medulloblastoma and prostate carcinomas. ${ }^{7,8,11}$ Therefore, we first evaluated whether $\mathrm{CD}_{133^{+}}$cancer cells could be found within lung tumors. Immunohistochemical analysis performed on patient-derived tumor sections indicated the existence of rare $\mathrm{CD}_{133^{+}}$cells in lung tumors. $\mathrm{CD}_{133^{+}}$cells were generally infrequent but consistently detectable in all the different patients analyzed, with some areas rich of positive cells (Figure 1a). In contrast, such CD133-expressing cells were not detectable by immunohistochemistry in control lung tissue specimens (Figure 1a). To quantify more precisely the percentage of $\mathrm{CD}_{133^{+}}$cells in lung tumors, we analyzed enzymatically dissociated cancer tissues derived from both NSCLC and SCLC by flow cytometry. The three major types of NSCLC were examined, including SCC, AC and LCC. The latter displays a particular heterogeneity. Therefore, the analysis was restricted to one of the most frequent histological variant, the large cell neuroendocrine carcinoma (LCNEC). These experiments confirmed that the percentage of $\mathrm{CD}_{13}{ }^{+}$cells was extremely low, with a few exceptions, but consistently higher than in normal lung tissue (Figure $1 \mathrm{~b}$ and Table 1). No correlation was observed between the percentage of $\mathrm{CD}_{133^{+}}$cells and the tumor subtype. As for colon carcinoma, ${ }^{14}$ the vast majority of $\mathrm{CD}_{133^{+}}$cells in NSCLC a

Lung cancer

Control lung
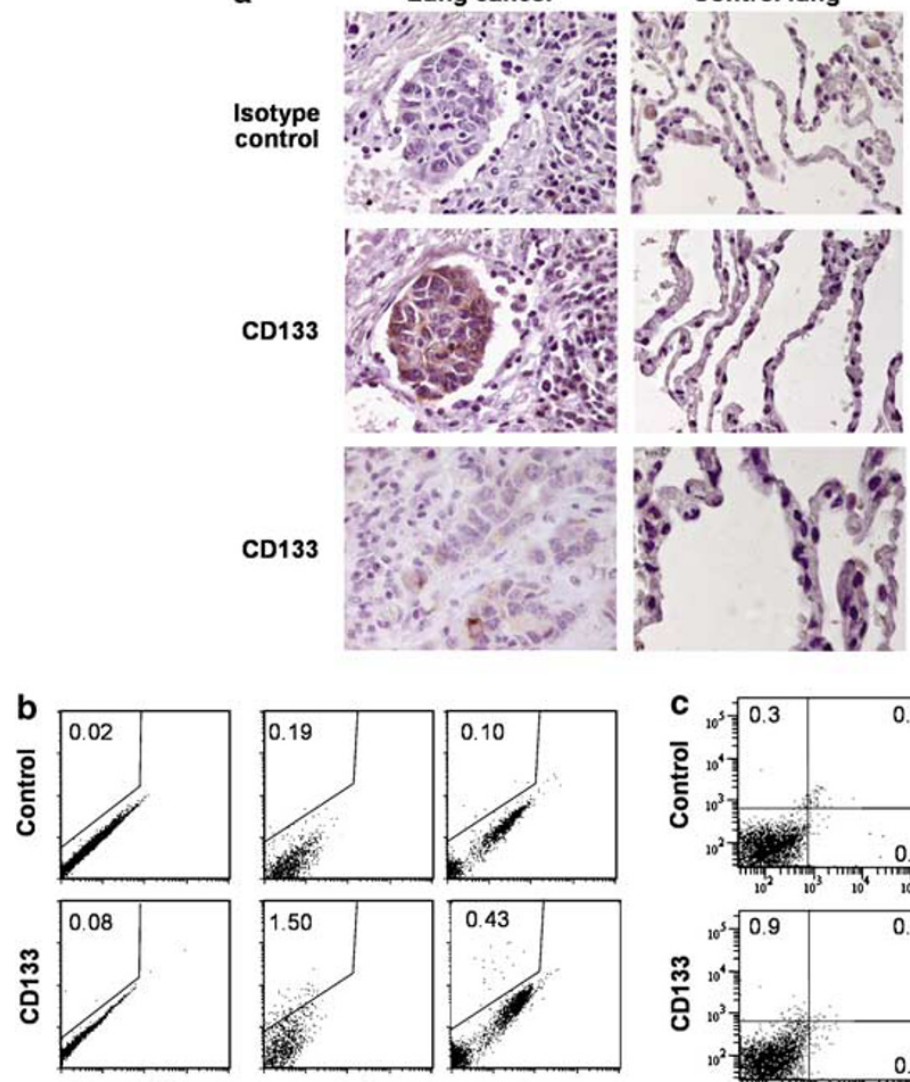

Control lung
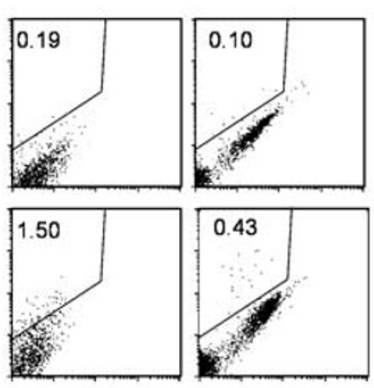

Lung cancer

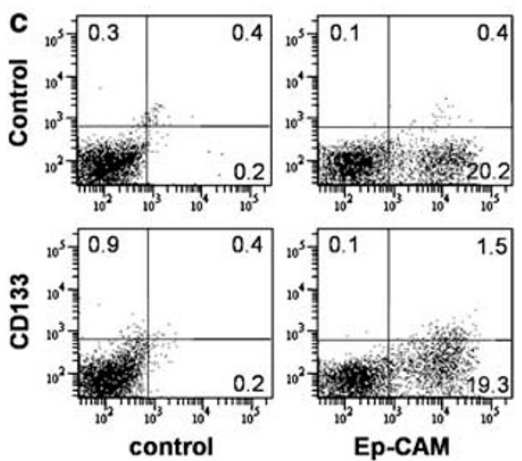

Figure 1 A population of $\mathrm{CD}_{133^{+}}$tumor cells is present in lung cancer. (a) Immunohistochemistry for $\mathrm{CD} 133$ shows a variable number of positive cells within patientderived SCC specimens (lung cancer) and absence of $\mathrm{CD}_{133^{+}}$cells within control lung tissue (control lung) derived from normal tissue samples surrounding the tumor of the same patient (right bottom) and non-oncological patients (up and middle right panels). Standard morphology of normal lung tissues is clearly visible (right panels). Control antibody staining on cancer and control samples is reported (isotype control). Cells were counterstained with hematoxylin. (b) Flow cytometry analysis of CD133 in one representative case of control lung tissue and two cases of freshly dissociated lung cancer samples. Percentages of $\mathrm{CD} 133^{+}$cells are indicated in control antibody (control) and specific antibody (CD133)-stained samples. (c) Flow cytometry analysis of freshly dissociated lung cancer cells double stained with CD133 PE and Ep-CAM FITC 
Table 1 Case description and lung tumor sphere formation

\begin{tabular}{|c|c|c|c|c|c|}
\hline Sample & $\begin{array}{l}\text { Patient } \\
\text { (sex/age, years) }\end{array}$ & $\begin{array}{l}\text { Tumor } \\
\text { subtype }\end{array}$ & TNM stage/grading & $\begin{array}{c}\text { CD133 } \\
\text { expression (\%) }\end{array}$ & $\begin{array}{l}\text { Sphere } \\
\text { formation }\end{array}$ \\
\hline 1 & $\mathrm{M} / 73$ & $A C$ & pT2pN2pMX(IIIA)/G3 & 1.1 & No \\
\hline 2 & $\mathrm{M} / 64$ & $A C$ & pT2pN1pMX(IIB)/G2 & 0.32 & No \\
\hline 3 & $\mathrm{M} / 78$ & $A C$ & pT2pN2pMX(IIIA)/G3 & 0.98 & Yes \\
\hline 4 & $\mathrm{~F} / 50$ & $A C$ & pT2pNOpMX(IB)/G2 & 0.33 & No \\
\hline 5 & $\mathrm{~F} / 68$ & $A C$ & pT1pN0pMX(IA)/G3 & 0.45 & No \\
\hline 6 & $\mathrm{M} / 78$ & $A C$ & pT1pNXpMX/G1 & 0.36 & No \\
\hline 7 & $\mathrm{M} / 77$ & $\mathrm{AC}$ & pT1pN0pMX(IA)/G1 & 0.44 & No \\
\hline 8 & $\mathrm{~F} / 57$ & $\mathrm{AC}$ & pT2pN0pMX(IB)/G1 & 0.35 & No \\
\hline 9 & $M / 68$ & $\mathrm{AC}$ & pT3pNOpMX (IIB)/G3 & 1.1 & Yes \\
\hline 10 & $\mathrm{M} / 70$ & SCC & pT2pN2pMX(IIIA)/G2 & 7 & Yes \\
\hline 11 & $\mathrm{M} / 57$ & SCC & урT3pNOpMX(IIB)/G2 & 1.7 & No \\
\hline 12 & $\mathrm{M} / 65$ & SCC & pT2pN0pMX(IB)/G2-3 & 0.35 & No \\
\hline 13 & $\mathrm{M} / 73$ & SCC & pT2pN0pMX(IB)/G3 & 1 & No \\
\hline 14 & $\mathrm{M} / 75$ & SCC & pT2pNOpMX(IB)/G3 & 1.13 & No \\
\hline 15 & $\mathrm{M} / 65$ & SCC & pT4pN2pMX(IIIB)/G3 & 22 & Yes \\
\hline 16 & $\mathrm{M} / 57$ & SCLC & pT1pN2pMX(IIIA) & 0.59 & Yes \\
\hline 17 & $\mathrm{~F} / 72$ & SCLC & pT3pN2pMX(IIIA) & 2.9 & Yes \\
\hline 18 & $\mathrm{M} / 57$ & LCNEC & pT2pN0pMX(IB)/G3 & 0.72 & No \\
\hline 19 & $\mathrm{M} / 63$ & LCNEC & pT2pN2pMX(IIIA)/G3 & 3.5 & Yes \\
\hline
\end{tabular}

Cells from freshly dissociated lung cancer tissues of four different subtypes (AC, adenocarcinoma; LCNEC, large cell neuroendocrine carcinoma; SCC, squamous cell carcinoma; SCLC, small cell lung carcinoma;) were analyzed by flow cytometry for CD133 expression. The ability of the same samples to generate lung cancer spheres in vitro was evaluated by prolonged culture in growth factor-containing serum-free medium. Tumor tissue of case 16 was obtained from a lymph node metastasis

expressed the epithelial antigen (epithelial cell adhesion molecule; Ep-CAM), suggesting that the detection of such $\mathrm{CD}_{133^{+}}$population in lung carcinoma is due to the increased number of undifferentiated epithelial cells within the tumor (Figure 1c). Both flow cytometry and immunohistochemistry analysis were performed using two different anti-CD133 antibodies, which gave rise to similar results (data not shown).

In view of recent findings indicating an increase in bronchoalveolar stem cell number following naphthaleneinduced mouse lung injury, ${ }^{16}$ we evaluated the number of CD133-positive cells in normal and naphthalene-treated mice. In accordance with the human data, flow cytometry analysis showed a minimal expression of CD133-positive cells in normal lung, which increased significantly 7 days following naphthalene treatment (Supplementary Figure 1a). Similarly, mouse CD133 RNA was barely detected in control mouse lung cells, but increased significantly in injured lungs (Supplementary Figure 1b). Thus, $\mathrm{CD}_{133^{+}}$cells are extremely rare in normal lung tissue, while representing a small but significant fraction in pathological conditions where stem and progenitor cells are supposed to increase, such as in tissue regeneration and cancer.

Generation of CD133 ${ }^{+}$lung cancer spheres from SCLC and NSCLC. To determine whether lung cancer CD133 ${ }^{+}$ cells can expand and generate long-term cultures in vitro, freshly dissociated tumor cells from SCC, AC, LCNEC and SCLC were cultured at low density in serum-free medium containing epidermal growth factor (EGF) and basic fibroblast growth factor (FGF). As we previously showed, these culture conditions allowed the selection of undifferentiated colon or glioblastoma cancer stem and progenitor cells, while serum-dependent differentiated tumor cells and non-transformed accessory cells were negatively selected. ${ }^{14,22}$ Exposure of lung cancer cells to such growth factors in the absence of serum allowed the selective growth of $\mathrm{CD}_{133^{+}}$cells, which increased in number (Figure 2a) and gradually became a homogeneous population of $\mathrm{CD}_{133^{+}}$undifferentiated cells expressing the carcinoembryonic antigen (CEA) but not hematopoietic or endothelial markers (Figure 2b). After approximately 1 or 2 months, these cell cultures became exclusively formed by cellular clusters resembling the so-called 'tumor spheres' (Figure 2c). In contrast, CD133- cells did not acquire CD133 expression and die within 2-3 weeks of cultures in standard stem cell medium (data not shown). Cells from all the different subtypes of lung cancer spheres consistently expressed high levels of CD133 and low levels of CD34 (Figure 2c). NSCLC (AC, SCC and LCNEC) spheres expressed considerable amounts of Ep-CAM, but not of cytokeratins (CKs) (Figure 2c), which are acquired during epithelial cell differentiation. ${ }^{23}$ Likewise, undifferentiated cells of both SCLC and LCNEC expressed very low levels of neural cell adhesion molecule (N-CAM), a key marker of neuroendocrine tumors (Figure 2c). As for other tumor types, we obtained the formation of long-term growing spheres from a subset of tumors, suggesting that only 7 of the 19 lung tumors analyzed contained $\mathrm{CD}_{133^{+}}$cells able to grow in such culture conditions (Table 1).

$\mathrm{CD}_{133^{+}}$lung cancer spheres were subsequently analyzed for markers expressed in human side population cells (BCRP1) and in mouse alveolar (SP-C) or Clara cells (CC-10). PCR analysis showed that lung cancer spheres from all the subtypes of lung cancer expressed BCRP1, while SSC spheres expressed CC-10 and SP-C, and only one of the two AC-derived stem cell samples expressed SP-C (Supplementary Figure $2 \mathrm{a}-\mathrm{c}$ ).

Since mouse bronchoalveolar stem cells express surfactant protein C (SP-C), we investigate further the stem cell population not expressing this marker. Interestingly, we observed that the progeny of such $\mathrm{AC}$ spheres acquired SP-C during differentiation 
a
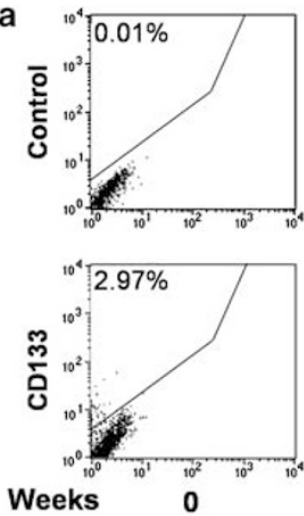

b

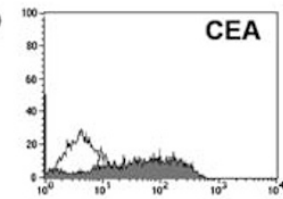

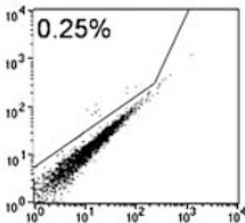

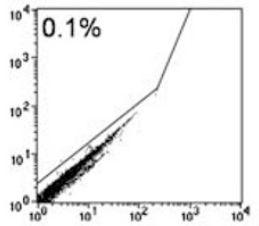

C
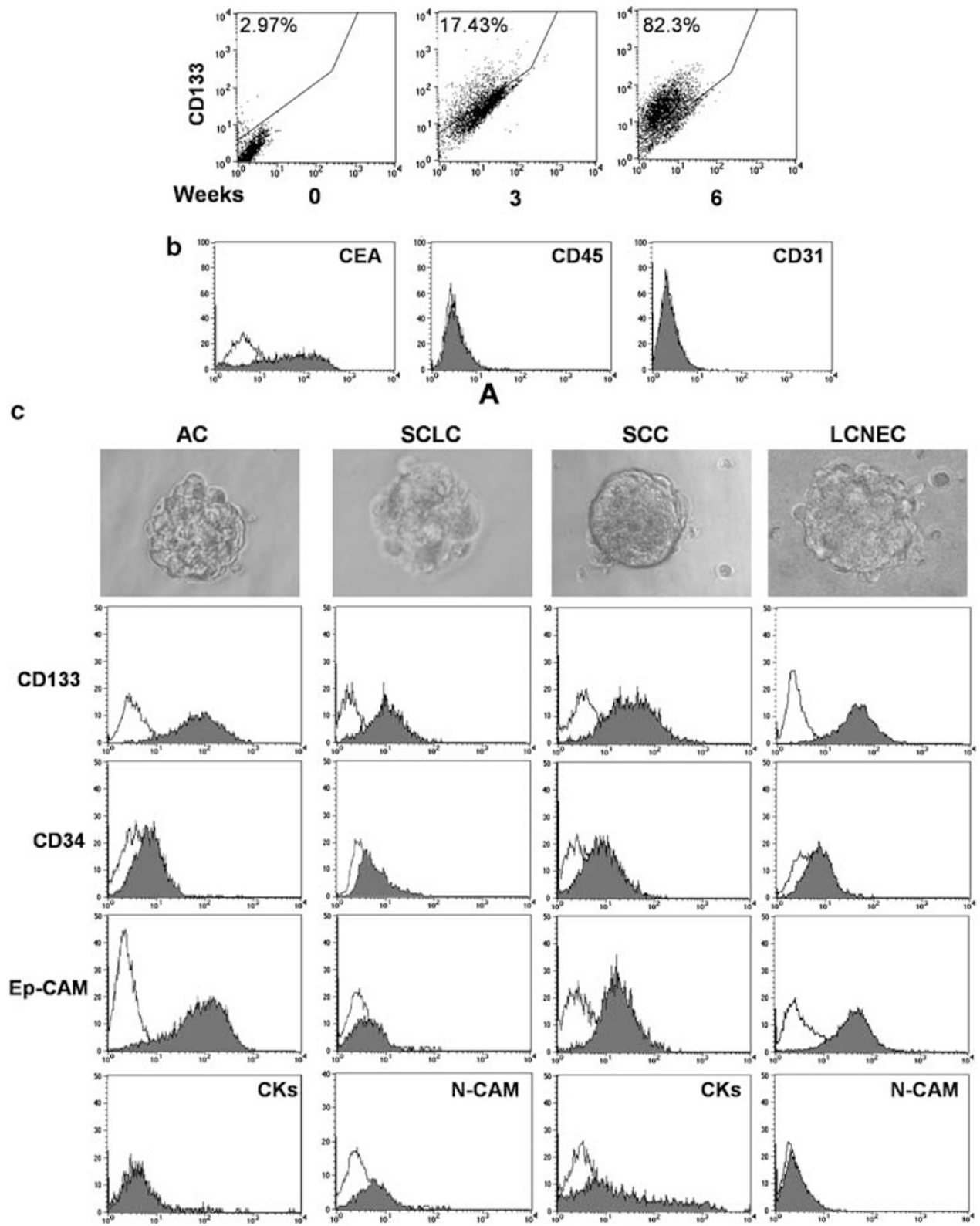

Figure 2 Establishment of lung cancer spheres containing CD133+ cells. (a) Flow cytometry detection of CD133 in freshly dissociated lung tumor cells or in the same cells cultured for the indicated times. Upper panels represent control antibody analysis of the corresponding CD133-stained cells in bottom panels. (b) Flow cytometric detection of carcinoembryonic (CEA), hematopoietic (CD45) or endothelial (CD31) antigens in lung cancer sphere-forming cells. All subtypes of lung cancer spheres displayed a similar expression for these antigens. (c) Phase-contrast photographs of lung cancer spheres obtained from the indicated tumor subtypes (upper panels) and flow cytometry detection of the indicated antigens in the corresponding cells (lower panels). Gray histograms correspond to specific antibody staining, white histograms represent negative control antibodies

(Supplementary Figure 2c), indicating that SP-C-negative immature cells may generate a differentiated progeny expressing markers typical of AC cells. In addition, lung cancer spheres expressed the embryonic cell markers Oct3/4 and Nanog
(Supplementary Figure 2d), whose expression has been reported in other cancer stem cells and in mouse pulmonary stem/progenitor cells, ${ }^{24,25}$ confirming the immature phenotype of the $\mathrm{CD}_{133^{+}}{ }^{\text {cancer population. }}$ 


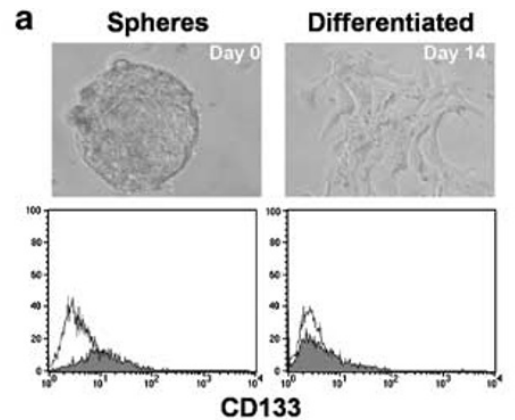

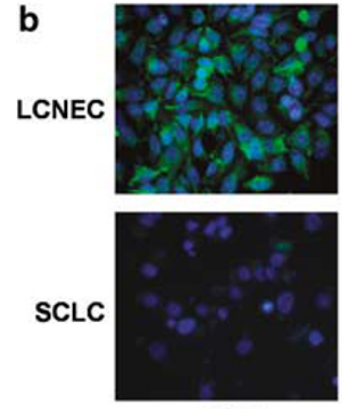

CKs
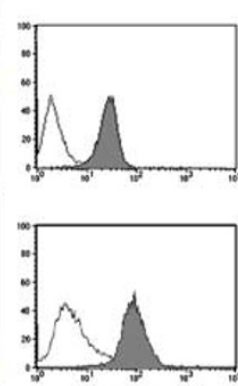

N-CAM

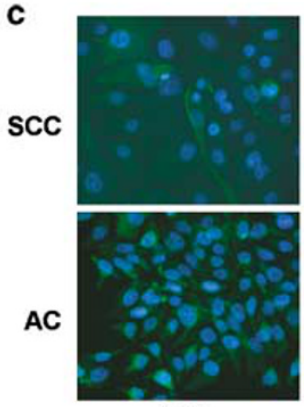

CKs

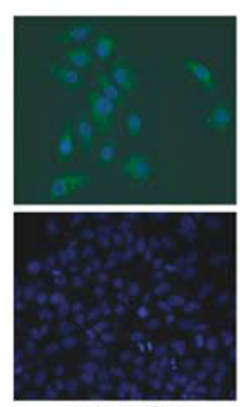

HMW-CKs

Figure 3 In vitro differentiation potential of lung cancer spheres. (a) Microscopical analysis of lung cancer spheres grown as undifferentiated cells (spheres) or under differentiative conditions for 2 weeks (differentiated). CD133 expression in the corresponding spheres and sphere-derived adherent progeny (lower panel). (b, c) Expression of lung cell antigens in sphere-derived differentiated progeny assessed by immunofluorescence staining and confocal (CKs and HMW-CKs) or flow cytometry (N-CAM) analysis

\begin{abstract}
Lung cancer spheres generate a differentiated progeny with phenotypic features of lung cancer cells. We next analyzed the in vitro differentiation potential of lung cancer spheres. In the presence of serum or specific medium for primary lung cell cultures, lung cancer spheres adhered to the plastic and acquired the typical morphologic features of differentiated cells (Figure 3a). Both spheres and differentiated cells expressed CEA (results not shown), while the CD133 antigen was lost during differentiation, confirming its specific expression in undifferentiated cells (Figure 3a). In contrast, the typical antigens found in the corresponding original tumors were gradually acquired after 1 week of culture. Specifically, we observed a considerable induction of N-CAM expression in the progeny of both large and small neuroendocrine lung cancer spheres (Figure $3 b$ ). Low and medium molecular weight CKs were detected in all NSCLC (AC, SCC and LCNEC) cells, while the expression of high molecular weight CKs (HMW-CKs) was induced only in SCC, indicating that lung cancer spheres are committed to produce a progeny of differentiated cells with phenotypic features of lung tumor cells (Figure $3 b$ and $c$ ). Thus, like cancer stem cells from other tumors, ${ }^{8,9,11,14,15}$ lung cancer spheres are composed by undifferentiated cells able to expand in the presence of EGF and basic FGF, but readily generating large and differentiated cells closely resembling the main cellular population of the original tumor under appropriate conditions.
\end{abstract}

Lung cancer spheres are tumorigenic in vivo and reproduce the human tumor. We next evaluated the tumorigenic potential of lung cancer $\mathrm{CD} 133^{+}$cells through subcutaneous injection of lung sphere cells mixed with growth factor-reduced matrigel in severe combined immunodeficiency (SCID) mice. The injection of as low as $10^{4} \mathrm{CD} 133^{+}$cells consistently resulted in the growth of tumor xenografts with morphological features closely resembling the original tumor, as shown by hematoxylin and eosin staining (Figure 4). In addition, immunohistochemistry analysis showed that the immature malignant cells isolated from the different subtypes of lung cancers could generate mouse xenografts with antigen expression highly similar to the original tumor. Specifically, SCC displayed strong positivity for HMW-CKs (Figure 4a), while the other NSCLCs expressed CKs (Figure $4 b$ and $c$ ). Likewise, N-CAM was expressed by small and large cell neuroendocrine tumors, and SCLC xenografts expressed chromogranine A (ChrA), another diagnostic marker for neuroendocrine tumors (Figure $4 \mathrm{c}$ and $\mathrm{d}$ ). Complete similarity between patient tumor and mouse xenograft was found for all antigens examined, demonstrating that tumor spheres could effectively reproduce the human disease in the mouse. Importantly, mouse xenograft-derived cells could be serially transplanted in secondary and tertiary recipients, readily generating tumors with similar morphological and antigenic pattern (data not shown).

Lung cancer $\mathrm{CD} 133^{+}$cells loose the self-renewal and tumorigenic potential upon differentiation. To confirm that lung cancer is initiated by a population of stem-like cells, we compared the growth potential of undifferentiated and 

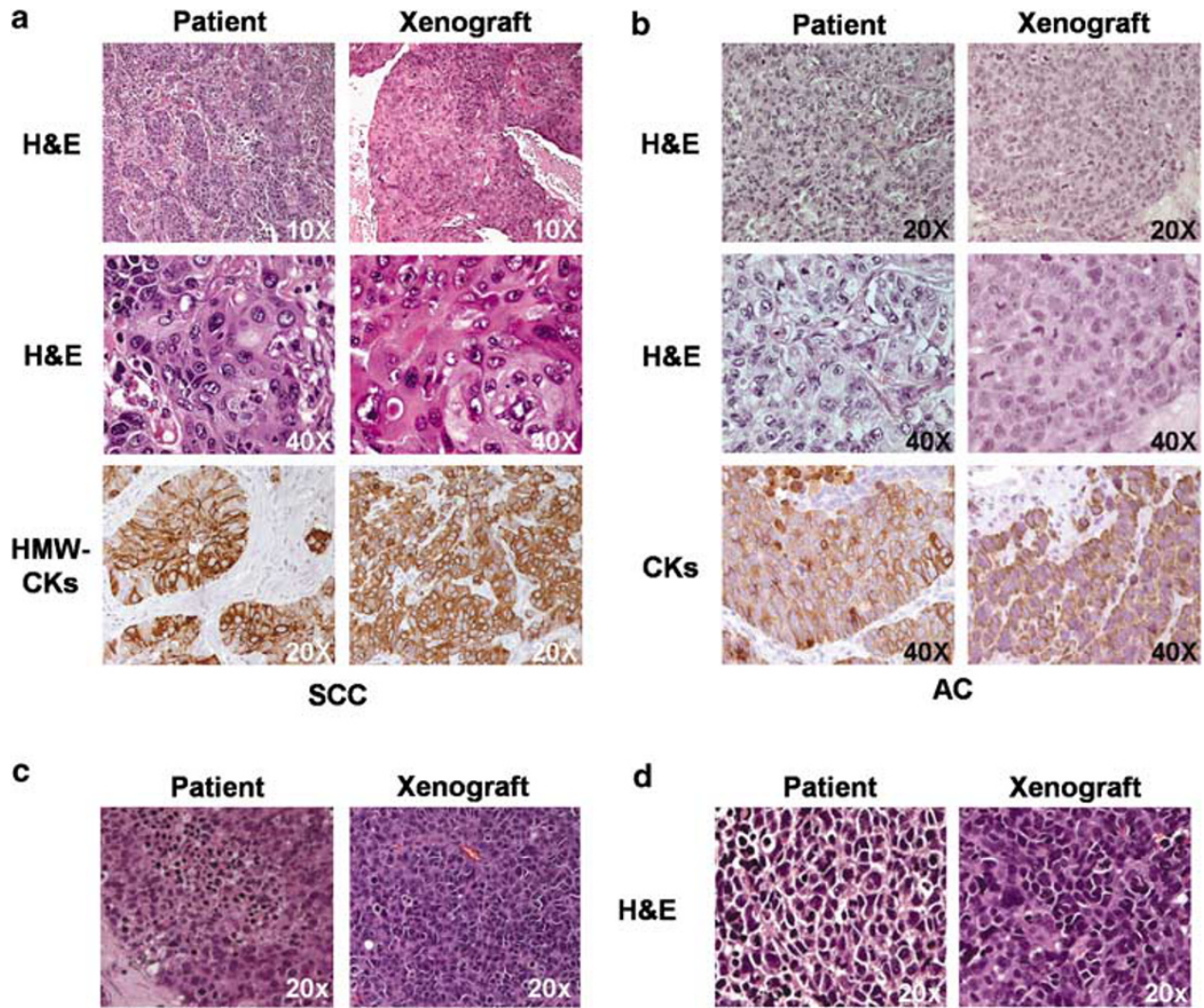

d
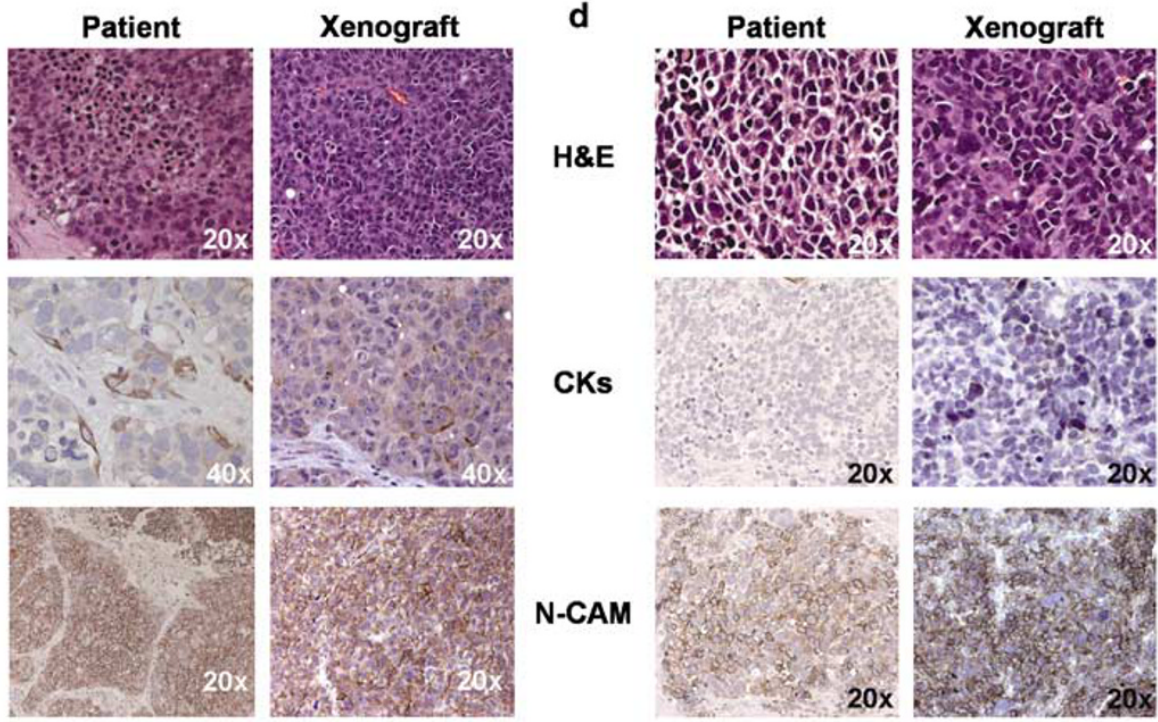

LCNEC

Chr A

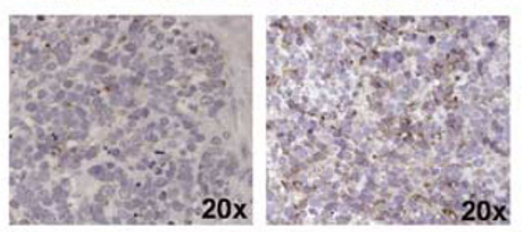

SCLC

Figure 4 SCC (a), AC (b), LCNEC (c) and SCLC (d) spheres are tumorigenic and reproduce the human tumor in immunocompromised mice. Hematoxylin and eosin $(\mathrm{H} \& \mathrm{E})$ or immunohistological staining for the indicated antigens performed on tumor specimens derived from parental tumor (patient) or from tumors generated by subcutaneous injection of lung cancer spheres in SCID mice (xenograft). The original magnification for each histological comparison is shown. Data are representative of four independent experiments

differentiating cells. While lung cancer spheres displayed a stable exponential growth, differentiating tumor cells were able to proliferate for about 4 weeks before declining in number (Figure 5a), suggesting that high proliferation potential of $\mathrm{CD} 133^{+}$lung cancer cells was lost during differentiation. To rule out the possibility that such a limited in vitro growth resulted from unfavorable culture conditions, we compared the tumorigenic potential of undifferentiated and differentiated cells in immunodeficient mice. While the subcutaneous injection of $10^{4}$ undifferentiated cells invariably produced tumor xenografts, a fivefold higher number of actively proliferating differentiated cells was unable to generate tumors in SCID mice (Figure 5b). Although cancer-derived spheres from single patients 

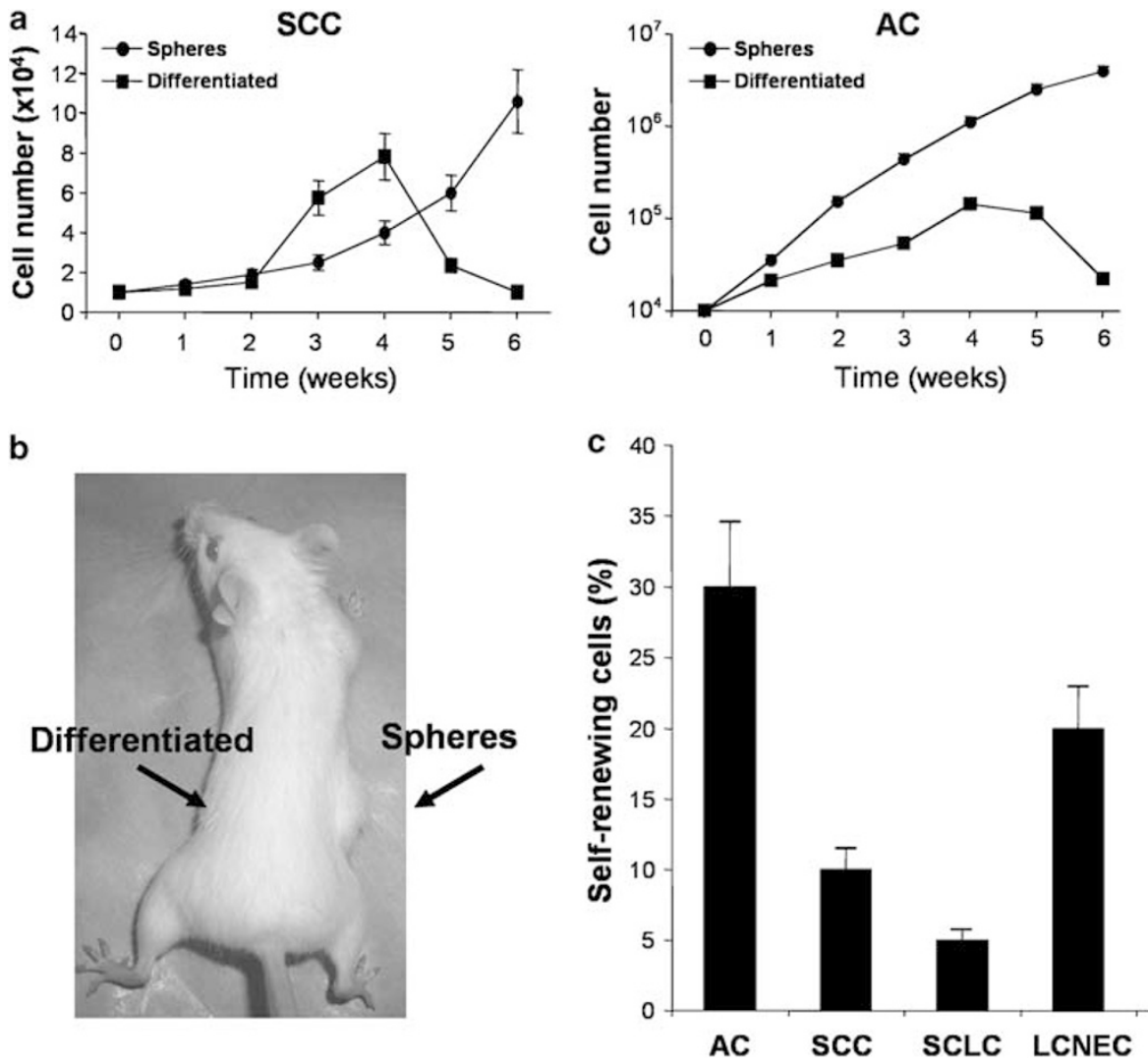

Figure 5 Self-renewal and tumorigenic potential of $\mathrm{CD} 133^{+}$lung cancer cells before and after differentiation. (a) Extended proliferative capacity of lung cancer-derived spheres in comparison with their differentiated progeny. Growth curve values were obtained by cell counting at the indicated time points. SCC- and AC-derived cells were used for the growth curve represented. (b) Tumorigenic potential of $10^{4}$ undifferentiated cells (spheres) as compared with $5 \times 10^{4}$ cells after 3 weeks of differentiation (differentiated). Cells were simultaneously injected into the right and left flank of the same mouse, and mouse picture was taken 3 months after injection. Data shown in (a, b) are representative of four independent experiments. (c) Number of self-renewing cells in lung cancer spheres. Data represent the percentage of long-term growing cells in lung cancer spheres plated as single cell per well. Self-renewing cells were defined based on the percentage of clones showing exponential growth as secondary tumor spheres for more than 5 months. Data are mean \pm S.D. of three independent experiments

displayed a variable growth rate, all the samples analyzed underwent virtually unlimited expansion in vitro. The cell number required for tumor formation in mice did not increase after several passages in culture, indicating that the stem cell potential was not lost with extended proliferation (results not shown). To determine the percentage of putative cancer stem cells in lung spheres, we evaluated by limiting dilution analysis the ability of single cells to auto-replicate and generate new spheres endowed with unlimited growth potential in secondary cultures. We found that lung cancer spheres contained a high percentage of self-renewing cells, which ranged from 5 to $30 \%$ (Figure $5 \mathrm{c}$ ). Thus, lung cancer stem-like cells can be unlimitedly expanded and maintained in culture as tumor spheres containing a considerable percentage of tumorigenic cells.

Prospective identification of lung cancer-initiating cells. The results obtained with the lung cancer spheres indicated that the tumorigenic cells in lung cancer are confined into the stem-like population expressing CD133 and Ep-CAM. To confirm that the lung cancer tumorigenic population expresses CD133, we directly purified the $\mathrm{CD}_{133^{+}}$and $\mathrm{CD} 133^{-}$cancer cells dissociated from lung tumor specimens. The use of Ep-CAM as secondary marker allowed us to compare the two cancer cell populations without the presence of contaminant stromal, endothelial and hematopoietic cells. Cells were labeled with PE-conjugated anti-CD133/1 antibody and fluorescein isothiocyanate (FITC)-conjugated anti-BerEP 4 (Ep-CAM) and purified by fluorescence-activated cell sorting (FACS). Purity of isolated $\mathrm{CD}_{133^{+}}$and $\mathrm{CD}_{133^{-}}$cell populations was evaluated by staining with allophycocyanin (APC)conjugated anti-CD133/2 antibody, directed against a different epitope of the CD133 protein (Figure 6a). The injection of $1 \times 10^{4} \mathrm{CD}_{133^{+}}$cells was able to consistently generate tumor xenografts upon subcutaneous injection into SCID mice, while a 10-fold higher number of CD133cells was not tumorigenic in the same animals (Figure 6b). Tumor xenografts generated by freshly isolated $\mathrm{CD} 133^{+}$ cells or lung cancer spheres equally reproduced the original tumor at histological examination, while displaying a similar number of $\mathrm{CD}_{133^{+}}$cells in the tumor mass as compared with the parental tumor (Figure 6a). These results confirmed that lung cancer-initiating cells are included in the CD133 ${ }^{+}$ population and that $\mathrm{CD} 133^{+}$cells expanded in vitro retained a similar tumorigenic potential and may be exploited to generate the original tumor in experimental models of lung cancer. 

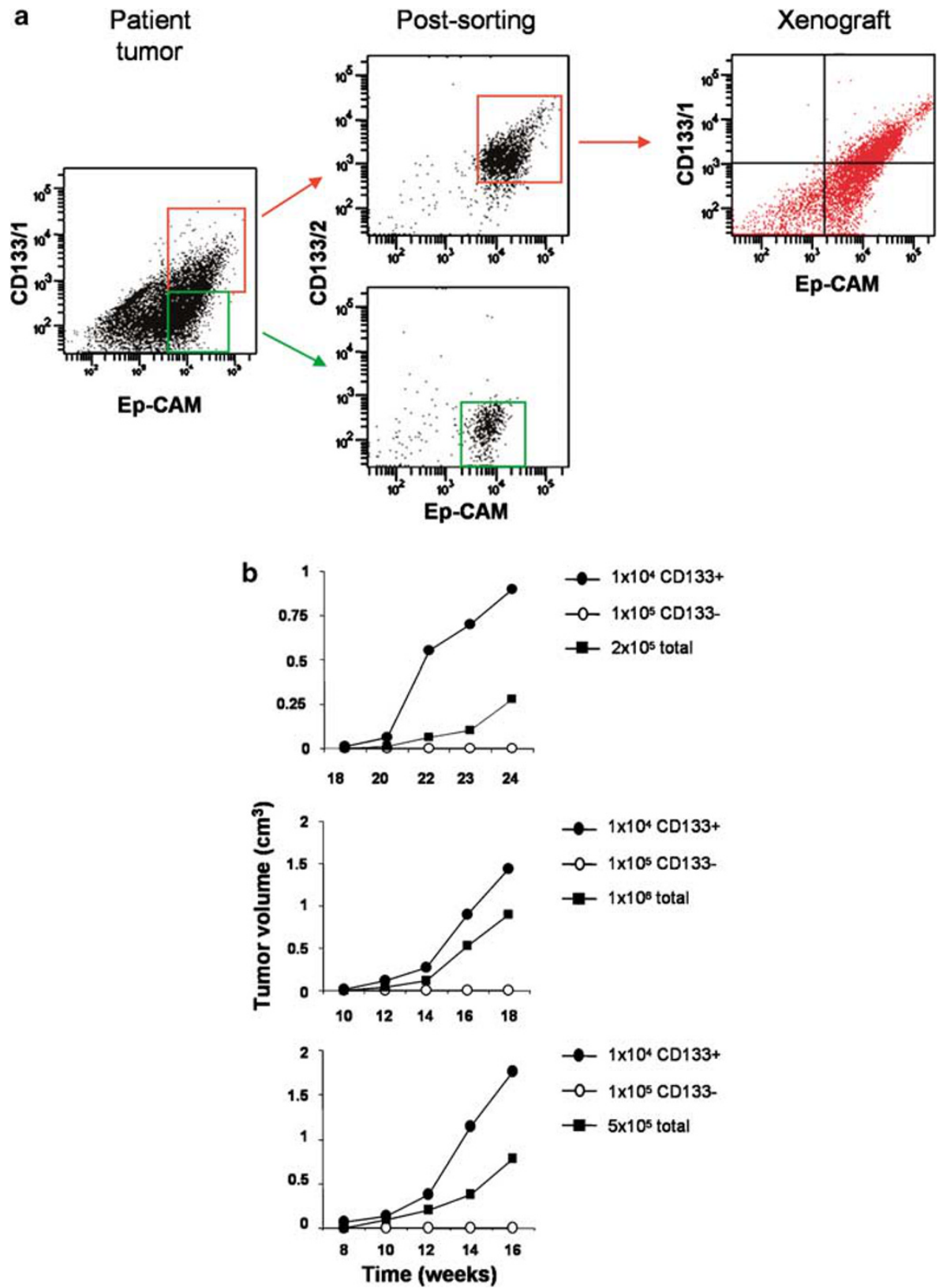

Figure 6 Tumorigenic potential of freshly isolated CD133+ lung cancer cells. (a) Cytofluorimetric cell sorting of double-labeled CD133/1-PE/Ep-CAM-FITC lung cancer cells (patient tumor), reanalyzed shortly after the sorting (post-sorting) and following mouse xenograft dissociation (xenograft). (b) Growth rate of mouse xenografts generated after subcutaneous injection of lung cancer cells from case no. 15 (top), no. 10 (middle) and no. 3 (bottom), unseparated (total) or purified by flow cytometry sorting as in (a)

Lung cancer stem cells are resistant to conventional chemotherapy. The establishment of exponentially growing lung cancer stem cell cultures may allow the direct evaluation of the cytotoxic activity of antineoplastic agents on the cells responsible for tumor growth and spreading, which represent the optimal cellular target for successful therapies. Therefore, we investigated the cytotoxic effect of the chemotherapeutic agents currently used in the clinical setting on lung cancer spheres. Cisplatin, etoposide, paclitaxel and gemcitabine were used at doses comparable with the higher plasma levels reached in treated lung cancer patients. Since in preliminary experiments these cells proved to be rather resistant to chemotherapeutic drugs, viability of lung cancer stem cells was evaluated after 5 days of treatment. Cisplatin and etoposide displayed a modest cytotoxic activity on all the different lung cancer stem cells examined, slightly higher in AC stem cells (Figure 7). Paclitaxel was partially effective on SCLC- and $\mathrm{AC}$-derived $\mathrm{CD}_{133^{+}}$cells only after prolonged exposure, which allowed to detect a more consistent cytotoxic activity on SCC and SCLC stem cells treated with gemcitabine (Figure 7 and data not shown). Thus, similarly to glioblastoma stem cells, ${ }^{22}$ lung cancer stem cells are resistant to chemotherapeutic drugs, in line with the poor therapeutic effect of conventional chemotherapy on lung cancer patients. 

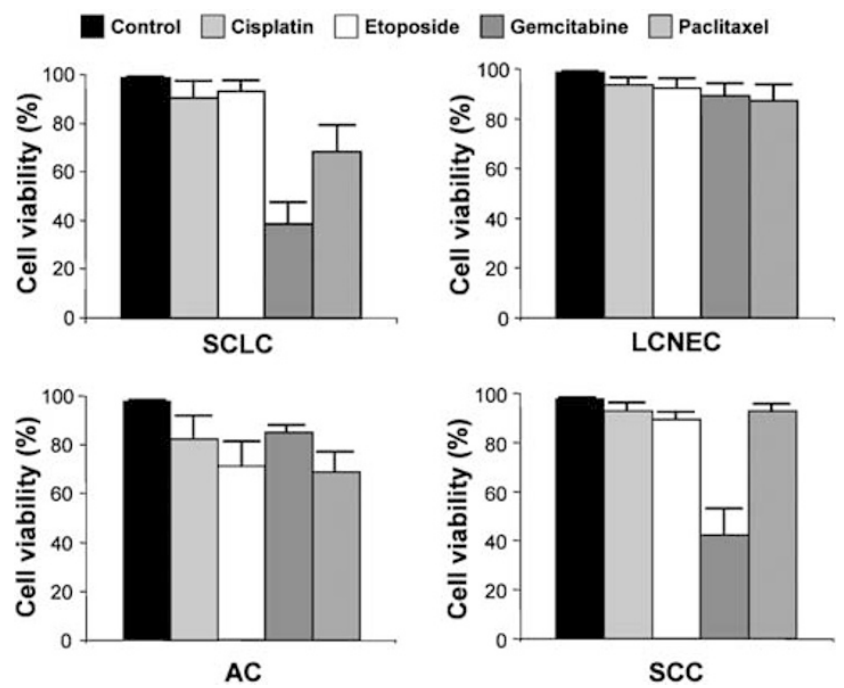

Figure 7 Chemotherapy resistance of lung cancer spheres. Cell viability of control or chemotherapy-treated lung cancer spheres. Gemcitabine $(250 \mu \mathrm{M})$, paclitaxel $(30 \mathrm{ng} / \mathrm{ml})$, cisplatin $(5 \mu \mathrm{g} / \mathrm{ml})$ and etoposide $(10 \mu \mathrm{g} / \mathrm{ml})$ were added to partially dissociated spheres of the indicated lung cancer subtypes and cell viability was evaluated after 5 days by MTT assay (Promega) and cell count. Data are mean of three independent experiments using two AC, two SCC, two SCLC and one LCNEC stem cell samples from different patients

\section{Discussion}

In spite of the variety of therapeutic attempts for the treatment of lung cancer, no major improvements in overall survival have been obtained so far. The identification and characterization of the tumorigenic population responsible for lung cancer formation and spreading may contribute to develop more effective therapies aiming at improving the prognosis of such severe condition.

Here, we identified the rare population of CD133 ${ }^{+}$cells as the lung cancer tumorigenic cell population. A low number of freshly isolated lung cancer $\mathrm{CD} 133^{+}$cells was able to reproduce the original tumor in immunocompromised mice, while the $\mathrm{CD}_{133^{-}}$cancer cell population was completely devoid of tumor-initiating activity. An unlimited number of these $\mathrm{CD}_{133^{+}}$cells were further obtained from SCLC and several NSCLC subtypes through the use of selective culture conditions that allowed their expansion as tumor spheres. Lung cancer spheres displayed undifferentiated cell phenotype revealed by CD133 expression and lack of lineagespecific lung cell markers, suggesting that lung cancer could be initiated and propagated by undifferentiated stem-like cells.

Lung cancer $\mathrm{CD} 133^{+}$cells displayed the ability to generate differentiated lung cancer cells under appropriate culture conditions, as demonstrated by simultaneous acquisition of lineage-specific markers and loss of CD133 expression. Such differentiated lung cancer cells were phenotypically very similar to the major cancer cell population present in the original tumor, indicating the existence of a precise hierarchical model for the formation of lung cancer tissue, based on the generation of a vast cell progeny by a small number of self-renewing undifferentiated cells.

Like the tumorigenic cells from other tumors, lung cancer $\mathrm{CD}_{133^{+}}{ }^{+}$cells were endowed with extensive proliferation and self-renewal potential, being able to grow as undifferentiated cells for more than 1 year without loosing the ability to reproduce the original tumor after transplantation in immunocompromised animals. The number of self-renewing cells in lung cancer spheres ranges from 5 to $30 \%$, as measured by clonogenic assays. Since CD133 expression is rather homogeneous in these cells, it is likely that lung cancer CD133 ${ }^{+}$ cells comprise two populations of cells with similar phenotype, but different potential: a tumorigenic population of stem-like cells able to self-renew and a non-tumorigenic population of progenitor/precursor cells with a limited proliferation potential that constitute the early progeny of putative lung cancer stem cells.

Our in vivo studies confirmed the high tumorigenic potential of lung cancer spheres. A low number of lung cancer CD133 ${ }^{+}$ cells was able to consistently generate tumor xenografts reproducing the original lung tumor both at morphologic and immunohistologic levels. This system may provide an excellent model to study lung cancer biology and its response to therapeutic approaches at preclinical level.

In view of results obtained with cells surviving lungdamaging agents, two subtypes of Clara cells have been identified in mice as the major lung-reparative populations resident in neuroepithelial bodies and at the bronchoalveolar duct junctions. Neuroepithelial bodies are scattered islands of amine- and peptide-containing vesicles that are released upon hypoxic stimulation. Surviving Clara cells of the neuroepithelial bodies can replenish both the neuroendocrine and epithelial cell populations. ${ }^{26}$ The second subtype of surviving Clara cells is located at the bronchoalveolar duct junction and seems to play a key role in the regeneration of the epithelial components of the bronchoalveolar structure. ${ }^{27}$ These reparative bronchoalveolar cells are able to self-renew and display all the features of a regional stem cell of the distal lung. ${ }^{16}$ Expression of K-ras promoted the transformation of mouse bronchoalveolar stem cells, which gave rise to lung adenocarcinomas after naphthalene treatment, suggesting that bronchoalveolar stem cells could be a target of tumor transformation in lung cancer. ${ }^{16}$ In this model, mouse lung cancer stem cells express Sca-1 and high levels of CD34. Sca-1 is not expressed in humans, while CD34 expression does not seem to characterize similar stem cell populations in mice and humans. ${ }^{28}$ Although there is not a full overlap between human and mouse stem cell antigens, human lung cancer $\mathrm{CD}_{133^{+}}$cells and mouse bronchoalveolar stem cells share to some extent the expression of the stem cell markers CD34, BCRP1, Oct4 and the absence of pan-hematopoietic (CD45) and -endothelial (CD31) antigens. ${ }^{16,24}$ Moreover, we observed that the rare $\mathrm{CD} 133^{+}$cells present in the mouse lung increase considerably following naphthalene-induced injury, in line with the possibility that CD133 may be expressed in mouse bronchoalveolar stem cells. In contrast, the bronchoalveolar stem cell and alveolar type 2 cell marker SP-C showed a variable expression in lung cancer spheres, reflecting the phenotypic variability of human lung cancer stem cells.

Lung cancer spheres contain a significant percentage of stem-like cells able to self-renew. However, they seemed to be lineage restricted, as indicated by the strict similarity between their progeny and the particular tumor subtype of the 
original tumor. Thus, it is theoretically possible that the genomic alterations contributing to the transformation of normal lung stem cells may force the differentiation toward a specific phenotype. Although the existence of rare tumors with a mixed phenotype suggests that lung stem cells can be the target of the oncogenic transformation, we cannot rule out the possibility that the tumorigenic lung cancer cells may derive from a transformed progenitor already committed to a particular lineage, rather than from a more primitive multipotent stem cell. Except for the common expression of CD133, the tumorigenic cells derived from different lung cancer subtypes display considerable variability in terms of proliferation rate, differentiation potential and number of clonogenic cells developing in long-term cultures, with major differences observed between neuroendocrine and nonneuroendocrine tumors. These observations suggest that the different types of lung cancers are maintained by aberrant immature cells committed to different lineages.

The current technology allows the establishment of longterm lung cancer stem cell cultures from about one-third of the tumors. Although there is obvious need to improve the in vitro conditions to generate these cultures from a higher number of tumors, the availability of unlimited numbers of cancerinitiating cells from all the major lung tumor subtypes may significantly contribute to the understanding of lung cancer biology. The considerable resistance to conventional chemotherapy displayed by lung cancer stem cells may explain the scarce therapeutic efficacy of current treatments. However, extensive phenotyping and characterization of CD133 ${ }^{+}$lung cancer cells may provide key information on relevant pathways to be targeted to increase the therapeutic response. Likewise, the possibility to detect the tumorigenic population may facilitate the development of new diagnostic and prognostic procedures. In this context, the use of preclinical models based on primary lung tumorigenic cells may represent a powerful tool to obtain new advances to be exploited in the clinical setting.

\section{Materials and Methods}

Isolation and culture of lung cancer spheres. Tumor samples were obtained in accordance with consent procedures approved by the Internal Review Board of Department of Laboratory Medicine and Pathology, Sant'Andrea Hospital, University La Sapienza, Rome. Surgical specimens were washed several times and left overnight in DMEM-F12 medium supplemented with high doses of penicillin/ streptomycin and amphotericin B to avoid contamination. Tissue dissociation was carried out by enzymatic digestion $(20 \mu \mathrm{g} / \mathrm{ml}$ collagenase II, Gibco-Invitrogen, Carlsbad, $\mathrm{CA}$ ) for $2 \mathrm{~h}$ at $37^{\circ} \mathrm{C}$. Recovered cells were cultured at clonal density in serum-free medium containing $50 \mu \mathrm{g} / \mathrm{ml}$ insulin, $100 \mu \mathrm{g} / \mathrm{ml}$ apo-transferrin, $10 \mu \mathrm{g} / \mathrm{ml}$ putrescine, $0.03 \mathrm{mM}$ sodium selenite, $2 \mu \mathrm{M}$ progesterone, $0.6 \%$ glucose, $5 \mathrm{mM}$ HEPES, $0.1 \%$ sodium bicarbonate, $0.4 \% \mathrm{BSA}$, glutamine and antibiotics, dissolved in DMEM-F12 medium (Gibco-Invitrogen) and supplemented with $20 \mu \mathrm{g} / \mathrm{ml}$ EGF and $10 \mu \mathrm{g} / \mathrm{ml}$ bFGF. Flasks non-treated for tissue culture were used to reduce cell adherence and support growth as undifferentiated tumor spheres. The medium was replaced or supplemented with fresh growth factors twice a week until cells started to grow forming floating aggregates. Cultures were expanded by mechanical dissociation of spheres, followed by re-plating of both single cells and residual small aggregates in complete fresh medium.

Differentiation of stem cell progeny. To obtain differentiation of lung cancer sphere-forming cells, stem cell medium was replaced with Bronchial Epithelial Cell Growth Medium (Cambrex, East Rutherford, NJ, USA) in tissue culture-treated flasks, to allow cell attachment and differentiation. The acquisition of differentiation markers and loss of stem cell markers were evaluated by flow cytometry or immunofluorescence as indicated above.

Flow cytometry and immunofluorescence. For flow cytometry, tumor spheres were dissociated as single cells, washed and incubated with the appropriate dilution of control or specific antibody. Antibodies used were PEconjugated anti-CD133/1, PE-conjugated anti-CD133/2 or APC-conjugated antiCD133/1 from Miltenyi Biotec (Bergisch Gladbach, Germany), anti-CD56/N-CAM (Neomarkers, Fremont, CA), FITC-conjugated anti-epithelial membrane antigen (Ep-CAM) (clone BerEP4), anti-human CKs, anti-CEA, FITC-conjugated anti-CD34 and anti-CD45 (all from DakoCytomation, Glostrup, Denmark), anti-CD31 (Becton Dickinson, Erembodegem, Belgium). After 45 min incubation, cells were washed or, where necessary, incubated with FITC- or PE-conjugated secondary antibodies for $30 \mathrm{~min}$ and washed again before analysis using either a FACScan or an LSRII flow cytometer (Becton Dickinson). For immunofluorescence studies, cells were grown on poly-D-lysine-coated glass coverslips, fixed with $2 \%$ paraformaldehyde for $20 \mathrm{~min}$ at $37^{\circ} \mathrm{C}$ and permeabilized with $0.1 \%$ Triton X-100/PBS for $3 \mathrm{~min}$ at room temperature before incubation with the specific or control antibody. Stained cells were visualized with an Olympus confocal microscope.

Immunohistochemistry on tumor sections. Immunohistochemistry was performed on formalin-fixed paraffin-embedded or frozen tissue. Paraffin sections $(5 \mu \mathrm{m})$ were dewaxed in xylene and rehydrated with distilled water. Sections were treated with heat-induced epitope retrieval technique using a citrate buffer ( $\mathrm{pH} 6)$. For $\mathrm{CD} 133$ detection, epitope retrieval technique was based on EDTA (pH 8). After peroxidase inhibition with $3 \% \mathrm{H}_{2} \mathrm{O}_{2}$ for $20 \mathrm{~min}$, the slides were incubated with the following antibodies: CD133/1 (Miltenyi Biotec), rabbit polyclonal anti-CD133 (Abcam, Cambridge, UK), low and medium molecular weight CKs, HMW-CKs and chromogranin A (DakoCytomation) or N-CAM (Neomarkers). The reaction was performed using Elite Vector Stain $A B C$ systems (Vector Laboratories) and DAB substrate chromogen (DakoCytomation), followed by counterstaining with haematoxylin.

Cell proliferation assays. Spheres were plated at $10000 \mathrm{cells} / \mathrm{ml}$ in growth medium supplemented with growth factors and after extended mechanical dissociation of culture aliquots, single cells were counted by Trypan blue exclusion once a week. Adherent differentiated cells were plated in six-well plates (10000 cells/well) and one well every week was used for cell count. To determine their self-renewal ability, lung cancer cells were seeded in 96-well plates containing a single cell per well. Shortly after seeding, single cell-containing wells were identified and analyzed for the ability to generate long-term-growing secondary spheres whose expansion was stable for more than 5 months.

Generation of subcutaneous lung cancer xenografts into SCID mice. For mice xenografts, cells were mechanically dissociated to obtain singlecell suspensions, diluted in growth factor-containing medium alone or mixed with matrigel before subcutaneous injection. Four-week-old female SCID or nude mice were used with similar results. Serial dilutions of cells (down to as low as $1 \times 10^{4}$ cells) were injected to evaluate the tumorigenic activity of lung cancer $\mathrm{CD} 133^{+}$ cells. Mice were monitored to check for the appearance of signs of disease, such as subcutaneous tumors or weight loss due to potential tumor growth in internal sites. When tumor diameters reached at least $5 \mathrm{~mm}$ in size, mice were killed and tumor tissue was collected, fixed in buffered formalin and subsequently analyzed by immunohistochemistry. Hematoxylin and eosin staining followed by immunohistochemical analysis were performed to analyze tumor histology and to compare mouse xenografts with patient tumors.

Cell sorting of $\mathrm{CD}^{-133^{+}}$and $\mathrm{CD}^{-133^{-}}$lung tumor cell populations. Single-cell suspensions from lung cancer specimens were prepared as described above and cryopreserved. After thawing, cells were double stained with PE-conjugated anti-CD133/1 antibody and FITC-conjugated anti-Ep-CAM antibody (clone BerEP4) and sorted with a FACS Aria (Becton Dickinson). Purity of the $\mathrm{CD}_{133^{+}}$and $\mathrm{CD}_{133^{-}}$cell populations was evaluated using APC-conjugated anti-CD133/2 antibody (Miltenyi Biotec). Isolated cells were injected as described for lung cancer sphere-forming cells, after the number of viable cells was assessed.

Chemotherapy resistance studies. Three thousand cells obtained from lung cancer sphere dissociation were plated in 96-well flat-bottom plates. 
Chemotherapic agents were added at the following final concentrations: gemcitabine $250 \mu \mathrm{M}$, paclitaxel $30 \mathrm{ng} / \mathrm{ml}$, cisplatin $5 \mu \mathrm{g} / \mathrm{ml}$ and etoposide $10 \mu \mathrm{g} / \mathrm{ml}$. Cell viability was evaluated after 5 days of treatment by both MTT (3-(4,5-dimethyl-2-thiazolyl)-2,5-diphenyl 2H-tetrazolium bromide) assay (Promega, Madison, WI, USA) and cell count by Trypan blue exclusion. Data represented are mean of three independent experiments performed with the two experimental procedures.

Acknowledgements. This study was supported by grants from the Italian Association for Cancer Research and Italian Health Ministry. We thank Enrico Duranti for assistance with immunohistochemical studies and Stefano Guida for general technical assistance.

1. Collins LG, Haines C, Perkel R, Enck RE. Lung cancer: diagnosis and management. Am Fam Physician 2007; 75: 56-63.

2. Petersen I, Petersen S. Towards a genetic-based classification of human lung cancer. Anal Cell Pathol 2001; 22: 111-121.

3. Jemal A, Siegel R, Ward E, Murray T, Xu J, Smigal C et al. Cancer statistics, 2006. CA Cancer J Clin 2006; 56: 106-130.

4. Pardal R, Clarke MF, Morrison SJ. Applying the principles of stem-cell biology to cancer. Nat Rev Cancer 2003; 3: 895-902.

5. Bonnet D, Dick JE. Human acute myeloid leukemia is organized as a hierarchy that originates from a primitive hematopoietic cell. Nat Med 1997; 3: 730-737.

6. Al-Hajj M, Wicha MS, Benito-Hernandez A, Morrison SJ, Clarke MF. Prospective identification of tumorigenic breast cancer cells. Proc Natl Acad Sci USA 2003; 100: 3983-3988.

7. Patrawala L, Calhoun T, Schneider-Broussard R, Li H, Bhatia B, Tang S et al. Highly purified CD44+ prostate cancer cells from xenograft human tumors are enriched in tumorigenic and metastatic progenitor cells. Oncogene 2006; 25: 1696-1708.

8. Singh SK, Clarke ID, Terasaki M, Bonn VE, Hawkins C, Squire J et al. Identification of a cancer stem cell in human brain tumors. Cancer Res 2003; 63: 5821-5828.

9. Singh SK, Hawkins C, Clarke ID, Squire JA, Bayani J, Hide T et al. Identification of human brain tumour initiating cells. Nature 2004; 432: 396-401.

10. Fang D, Nguyen TK, Leishear K, Finko R, Kulp AN, Hotz $S$ et al. A tumorigenic subpopulation with stem cell properties in melanomas. Cancer Res 2005; 65: 9328-9337.
11. Galli R, Binda E, Orfanelli U, Cipelletti B, Gritti A, De Vitis $S$ et al. Isolation and characterization of tumorigenic, stem-like neural precursors from human glioblastoma. Cancer Res 2004; 64: 7011-7021.

12. Prince ME, Sivanandan R, Kaczorowski A, Wolf GT, Kaplan MJ, Dalerba $P$ et al. Identification of a subpopulation of cells with cancer stem cell properties in head and neck squamous cell carcinoma. Proc Natl Acad Sci USA 2007; 104: 973-978.

13. Li C, Heidt DG, Dalerba P, Burant CF, Zhang L, Adsay V et al. Identification of pancreatic cancer stem cells. Cancer Res 2007; 67: 1030-1037.

14. Ricci-Vitiani L, Lombardi DG, Pilozzi E, Biffoni M, Todaro M, Peschle $\mathrm{C}$ et al. Identification and expansion of human colon-cancer-initiating cells. Nature 2006; 445: 111-115.

15. O'Brien CA, Pollett A, Gallinger S, Dick JE. A human colon cancer cell capable of initiating tumour growth in immunodeficient mice. Nature 2006; 445: 106-110.

16. Kim CF, Jackson EL, Woolfenden AE, Lawrence S, Babar I, Vogel S et al. Identification of bronchioalveolar stem cells in normal lung and lung cancer. Cell 2005; 121: 823-835.

17. Berns A. Stem cells for lung cancer? Cell 2005; 121: 811-813.

18. Otto WR. Lung epithelial stem cells. J Pathol 2002; 197: 527-535

19. Giangreco A, Groot KR, Janes SM. Lung cancer and lung stem cells: strange bedfellows? Am J Respir Crit Care Med 2006; 175: 547-553.

20. Summer R, Kotton DN, Sun X, Ma B, Fitzsimmons K, Fine A et al. Side population cells and Bcrp1 expression in lung. Am J Physiol Lung Cell Mol Physiol 2003; 285: L97-L104.

21. Summer R, Kotton DN, Sun X, Fitzsimmons K, Fine A. Translational physiology: origin and phenotype of lung side population cells. Am J Physiol Lung Cell Mol Physiol 2004; 287: L477-L483.

22. Eramo A, Ricci-Vitiani L, Zeuner A, Pallini R, Lotti F, Sette G et al. Chemotherapy resistance of glioblastoma stem cells. Cell Death Differ 2006; 13: 1238-1241.

23. Moll R. Cytokeratins as markers of differentiation in the diagnosis of epithelial tumors. Subcell Biochem 1998; 31: 205-262.

24. Ling TY, Kuo MD, Li CL, Yu AL, Huang YH, Wu TJ et al. Identification of pulmonary Oct-4+ stem/progenitor cells and demonstration of their susceptibility to SARS coronavirus (SARSCoV) infection in vitro. Proc Natl Acad Sci USA 2006; 103: 9530-9535.

25. Gibbs CP, Kukekov VG, Reith JD, Tchigrinova O, Suslov ON, Scott EW et al. Stem-like cells in bone sarcomas: implications for tumorigenesis. Neoplasia 2005; 7: 967-976.

26. Reynolds SD, Giangreco A, Power JH, Stripp BR. Neuroepithelial bodies of pulmonary airways serve as a reservoir of progenitor cells capable of epithelial regeneration. $A m \mathrm{~J}$ Pathol 2000; 156: 269-278

27. Giangreco A, Reynolds SD, Stripp BR. Terminal bronchioles harbor a unique airway stem cell population that localizes to the bronchoalveolar duct junction. Am J Pathol 2002; 161 173-182.

28. Osawa $\mathrm{M}$, Hanada $\mathrm{K}$, Hamada $\mathrm{H}$, Nakauchi $\mathrm{H}$. Long-term lymphohematopoietic reconstitution by a single CD34-low/negative hematopoietic stem cell. Science 1996; 273: 242-245.

Supplementary Information accompanies the paper on Cell Death and Differentiation website (http://www.nature.com/cdd) 